\title{
II. Efectos Fisiológicos de la Ventilación no Invasiva
}

\author{
ORLANDO DÍAZ P.*
}

\section{Physiological effects of noninvasive ventilation}

La ventilación no invasiva (VNI) se utiliza actualmente en una amplia variedad de condiciones que cursan con insuficiencia respiratoria aguda. En los últimos años, su indicación se ha ampliado incluso para prevenir la falla respiratoria post extubación. En todos estos casos, la VNI necesita lograr atenuar las dos características fisiopatológicas más importantes de la insuficiencia respiratoria aguda: a) la que la define clínicamente, esto es la alteración del intercambio pulmonar de gases; y b) la que acompaña invariablemente a la anterior, es decir, el aumento del trabajo respiratorio $\mathrm{y}$, por lo tanto, la disnea.

\section{Efectos fisiológicos en la EPOC}

Una de las alteraciones fisiopatológicas centrales de la enfermedad pulmonar obstructiva crónica (EPOC) es la presencia de limitación del flujo espiratorio (LFE), la que está presente incluso durante la respiración espontánea en pacientes con enfermedad avanzada. Las exacerbaciones de la EPOC empeoran bruscamente la LFE, debido a inflamación de la vía aérea pequeña y a la presencia concomitante de broncoconstricción ${ }^{1-3}$. Como consecuencia, en las exacerbaciones se puede observar hiperinflación pulmonar dinámica, aumento del trabajo respiratorio, alteraciones del intercambio pulmonar de gases y disfunción muscular respiratoria.

Cuando el tiempo espiratorio es insuficiente para permitir que el volumen de final de espiración (EELV; end-expiratory lung volume) alcance el volumen de reposo del sistema respiratorio, se produce hiperinflación pulmonar dinámica (HD) ${ }^{4,5}$. Durante las exacerbaciones, al empeorar la $\mathrm{LFE}^{3}$, el vaciamiento pulmonar se prolonga y la HD, generalmente presente en la eta- pa estable de la enfermedad avanzada, aumenta. Asimismo, el patrón respiratorio es rápido y superficial, limitando el tiempo espiratorio disponible para el vaciamiento pulmonar, y promueve mayor HD. El patrón respiratorio adoptado se ha interpretado como un intento para minimizar el gasto energético de respirar contra una carga inspiratoria extremadamente elevada ${ }^{6}$.

Debido a la HD, las presiones intratorácicas son positivas al final de la espiración, fenómeno conocido como presión positiva intrínseca de final de espiración (PEEPi o auto-PEEP). La PEEPi actúa como una carga inspiratoria umbral que los músculos inspiratorios deben vencer antes que el flujo inspiratorio pueda iniciarse. En pacientes clínicamente estables, la PEEPi oscila entre 6-9 $\mathrm{cmH}_{2} \mathrm{O}^{7,8}$, pudiendo aumentar a valores entre $13-20 \mathrm{cmH}_{2} \mathrm{O}$ durante las exacerbaciones, contribuyendo aproximadamente a un $60 \%$ del incremento del trabajo inspiratorio 9 . A diferencia del sujeto normal que respira en la porción inclinada de la curva presión-volumen del sistema respiratorio, en la exacerbación y como consecuencia de la $\mathrm{HD}$, el paciente con EPOC lo hace en una zona más elevada y plana, cercana a la capacidad pulmonar total. En esta zona se requieren grandes presiones inspiratorias para generar pequeños cambios de volumen. El aumento de la resistencia de la vía aérea por edema inflamatorio y broncoespasmo contribuye adicionalmente a aumentar el trabajo respiratorio.

Los músculos respiratorios se adaptan a la hiperinflación crónica, preservando su capacidad de generar fuerza durante la respiración tranquila ${ }^{10-12}$, pero dicha adaptación puede ser rápidamente sobrepasada si la HD aumenta bruscamente $^{13}$. La HD aguda acorta aún más los músculos inspiratorios, particularmente el diafragma, y provoca una debilidad muscular funcional ${ }^{6}$. * Profesor Asociado de Medicina, Departamento de Enfermedades Respiratorias, Pontificia Universidad Católica de
Chile. 
Asimismo, la exposición a estrés oxidativo y la activación local de proteasas daña las fibras musculares diafragmáticas, generando una disfunción muscular estructural ${ }^{14,15}$.

El efecto neto del aumento de la carga inspiratoria y la debilidad de los músculos inspiratorios es que el esfuerzo requerido para una inspiración a volumen corriente (VT) representa una fracción elevada de la fuerza máxima disponible. El estímulo del centro respiratorio está generalmente preservado en la EPOC y aumenta bajo situaciones de estrés, como son el ejercicio y las exacerbaciones ${ }^{16,17}$. Puede aumentar adicionalmente en presencia de hipoxemia, hipercapnia, acidosis y fiebre. Dado que la respuesta mecánica (es decir, el cambio de volumen) no aumenta en proporción al estímulo central, se produce un desacoplamiento neuromecánico. Esta disparidad entre estímulo central y respuesta mecánica como consecuencia de la HD aguda ha sido implicada en la génesis de la disnea observada durante el ejercicio ${ }^{18,19}$ y durante episodios inducidos de broncoconstricción ${ }^{20}$, por lo que es posible que juegue un rol similar en las exacerbaciones.

Es habitual que exista además hipoxemia y grados variables de hipercapnia y acidosis respiratoria. El empeoramiento de las relaciones $\dot{\mathrm{V}}_{\mathrm{A}} / \dot{\mathrm{Q}}$ explica gran parte de la hipoxemia observada ${ }^{21}$. Por otra parte, pese a que la ventilación minuto aumenta durante la exacerbación, resulta ineficaz para remover el $\mathrm{CO}_{2}$ ya que se produce a expensas de un aumento de la frecuencia respiratoria, mientras que el VT generalmente disminuye. En consecuencia, la hipercapnia reflejaría la incapacidad para aumentar el VT en presencia de un espacio muerto fisiológico fijo, como resultado del aumento de la LFE. Esta situación puede empeorar si las demandas metabólicas y la producción de $\mathrm{CO}_{2}$ aumentan.

La VNI, administrada usando ventilación con presión de soporte (PSV) y presión positiva de final de espiración (PEEP) ${ }^{22,23}$, asiste la inspiración en sincronía con el esfuerzo del paciente y reduce el trabajo respiratorio. Este efecto se debe a que la PEEP contrarresta el efecto de la PEEPi, disminuyendo el trabajo elástico estático, mientras que la PSV reduce el trabajo elástico y resistivo dinámico ${ }^{22-24}$. La consecuencia final es una disminución de las deflexiones de la presión esofágica, que reflejan la menor presión pleural necesaria para inspirar; de la presión transdiafragmática, que indica menor trabajo diafragmático; y del producto presión-tiempo de los músculos respiratorios, marcador indirecto del menor trabajo respiratorio ${ }^{22,23}$. La reducción del esfuerzo muscular se acompaña de un aumento del VT y una reducción de la frecuencia respiratoria, incrementándose el volumen minuto ${ }^{25}$.

La VNI es también capaz de revertir al menos en parte las anormalidades del intercambio de gases $^{22,25}$. Esta mejoría ha sido demostrada ampliamente en los casos en que la aplicación de VNI es exitosa. Habitualmente el efecto sobre los gases arteriales es evidente dentro de las primeras horas y se debe principalmente a un aumento de la ventilación alveolar, debido a que el patrón respiratorio se modifica desde uno rápido y superficial a uno más lento y profundo, sin cambios en las alteraciones $\dot{\mathrm{V}}_{\mathrm{A}} / \dot{\mathrm{Q}}^{25}$.

\section{Efectos fisiológicos en el edema pulmonar agudo cardiogénico}

La característica principal del edema pulmonar agudo cardiogénico (EPA) es un incremento rápido de la presión hidrostática de los capilares pulmonares, que aumenta la tasa de filtración transvascular de fluidos hacia el intersticio y los espacios alveolares. La cantidad neta de edema acumulado es determinada por el balance entre la tasa de filtración y la velocidad de remoción ${ }^{26,27}$.

En el EPA la distensibilidad del parénquima pulmonar disminuye ${ }^{28,29}$, mientras aumenta concomitantemente la resistencia de la vía aérea $^{30,31}$. El aumento de la resistencia, que no necesariamente se traduce en obstrucción bronquial clínica, podría deberse al menor volumen pulmonar; a broncoconstricción refleja, mediada por eferentes vagales; a estrechamiento de la vía aérea pequeña al competir por espacio en el manguito broncovascular; y también a compresión de las vías aéreas centrales ${ }^{30-33}$. La mayor resistencia de la vía aérea puede provocar LFE, generando $\mathrm{PEEPi}^{34}$. La consecuencia de estos fenómenos es un marcado aumento del trabajo respiratorio $^{29,35}$ y de la disnea ${ }^{36}$.

En el curso del EPA los músculos respiratorios deben generar grandes deflexiones negativas de la presión pleural para iniciar la inspiración y mantener un VT adecuado ${ }^{37}$. Desafortunadamente, estas presiones negativas agravan el edema, ya que incrementan tanto la precarga como la postcarga ventricular ${ }^{38,39}$. Esto se explica porque el llenado y vaciado cardíaco es determinado en parte por la diferencia de presión intracavitaria e intratorácica, que corresponde a la presión transmural $\left(\mathrm{P}_{\mathrm{TM}}\right)$. Dado que, como se ha dicho, la amplitud de las deflexiones inspiratorias es mayor que lo normal en los pacientes 
con edema pulmonar agudo, la $\mathrm{P}_{\mathrm{TM}}$ se encuentra aumentada ${ }^{39}$. Mientras mayor es la $\mathrm{P}_{\mathrm{TM}}$ durante la diástole, mayor es el llenado cardíaco (precarga). A su vez, mientras mayor es la $\mathrm{P}_{\mathrm{TM}}$ durante la sístole, mayor es el trabajo cardíaco (postcarga).

La aplicación de presión positiva continua de la vía aérea (CPAP) o de VNI con PSV y PEEP produce dos tipos de efectos benéficos en el EPA, mejorando la función respiratoria y promoviendo una mejoría de la función cardíaca. En efecto, al elevar la presión intratorácica, tanto la CPAP como la VNI disminuyen el cortocircuito intrapulmonar, mejoran la oxigenación y reducen la disnea ${ }^{40,41}$. Por otro lado, la CPAP reduce las deflexiones inspiratorias durante la sístole y consecuentemente disminuye la $\mathrm{P}_{\mathrm{TM}}$, por lo que cae el retorno venoso, reduciéndose la precarga de ambos ventrículos. A la vez, durante la diástole, la CPAP aumenta la presión intratorácica y pericárdica, lo que determina una disminución de la $\mathrm{P}_{\mathrm{TM}}$ y una reducción de la post carga ${ }^{42,43}$. La reducción de la precarga favorecería una resolución más rápida del edema, mientras que la menor pre y postcarga favorece la función ventricular y puede promover un aumento del gasto cardíaco. Se ha observado que el efecto sobre la precarga es similar en pacientes con disfunción sistólica y diastólica, mientras que el incremento del gasto cardíaco sólo se produce cuando la contractilidad está comprometida $^{40,41}$.

El empleo de PSV más PEEP induce beneficios fisiopatológicos similares a la CPAP ${ }^{44,45}$. Sin embargo, y a diferencia de la VNI, la CPAP no tiene un efecto directo sobre la ventilación alveolar. Esto explicaría que la VNI se asocie a una mejoría más rápida de la disnea y del intercambio gaseoso, particularmente cuando existe hipercapnia $^{46,47}$.

\section{Efectos fisiológicos en la injuria pulmonar aguda}

La injuria pulmonar aguda $\left(\mathrm{PaO}_{2} / \mathrm{FIO}_{2}\right.$ entre 200-300 $\mathrm{mmHg}$ ) y el síndrome de distrés respiratorio agudo $\left(\mathrm{PaO}_{2} / \mathrm{FIO}_{2}\right.$ menor de $\left.200 \mathrm{mmHg}\right)$ se definen por la presencia de hipoxemia refractaria, en asociación con infiltrados pulmonares bilaterales radiográficos, en presencia de una condición predisponente conocida, de origen pulmonar o extrapulmonar ${ }^{48}$. En ambos casos existe inflamación alveolar y aumento de la permeabilidad capilar, siendo sus consecuencias clínicas hipoxemia refractaria al empleo de altas concentraciones de oxígeno por cortocircuito intrapulmonar ${ }^{49}$, y aumento del trabajo respiratorio debido a una marcada disminución de la distensibilidad pulmonar ${ }^{50}$.

Sólo un estudio ha evaluado los efectos fisiológicos de la VNI en la injuria pulmonar aguda asociada a insuficiencia respiratoria hipoxémica ${ }^{51}$. El estudio incluyó 10 pacientes, en 7 de los cuales el factor predisponente había sido una neumonía. Se comparó el efecto a corto plazo de CPAP a $10 \mathrm{~cm} \mathrm{H}_{2} \mathrm{O}$ (CPAP 10) y dos combinaciones de VNI con presión de soporte: una con soporte inspiratorio de $10 \mathrm{cmH}_{2} \mathrm{O}$ y PEEP de $10 \mathrm{cmH}_{2} \mathrm{O}$ (PSV 10-10); otra con soporte inspiratorio de $15 \mathrm{~cm} \mathrm{H}_{2} \mathrm{O}$ y PEEP de $5 \mathrm{cmH}_{2} \mathrm{O}$ (PSV 15-5). En comparación con la respiración espontánea, la frecuencia respiratoria sólo disminuyó con el mayor nivel de soporte inspiratorio (PSV 15-5), mientras que la $\mathrm{PaO}_{2}$ mejoró con los mayores niveles de PEEP (CPAP 10 y PSV 10-10). Por su parte, el trabajo respiratorio no se redujo con CPAP, pero sí al emplear soporte inspiratorio, siendo el efecto mayor con PSV 15-5. En otras palabras, y como intuitivamente cabría esperar, en la insuficiencia respiratoria aguda existe un efecto sobre el esfuerzo inspiratorio que es aliviado por la presión de soporte inspiratorio y un efecto sobre la oxigenación, mediado por PEEP. Por lo tanto, sólo la VNI y no la CPAP es útil para modificar los trastornos fisiológicos asociados a esta condición.

\section{Efectos fisiológicos en el proceso de desconexión}

Los mecanismos que retrasan la discontinuación de la ventilación mecánica pueden ser muy variados $^{52}$, pero los más frecuentes son la persistencia de una carga inspiratoria inapropiadamente elevada en concomitancia o no con una menor fuerza muscular respiratoria que compense la carga. En otros pacientes, la presencia de disfunción miocárdica previamente conocida o desencadenada por la desconexión puede jugar un rol preponderante en el fracaso.

La presencia de una carga inspiratoria elevada ha sido demostrada en varios estudios fisiológicos que han incluido pacientes con y sin EPOC. El mismo proceso de desconexión puede aumentar la carga inspiratoria resistiva por efecto del tubo endotraqueal y/o la acumulación de secreciones traqueales ${ }^{53,54}$. En aquellos pacientes en los que fracasa la prueba de respiración espontánea se ha observado que el producto presión-tiempo del diafragma o las deflexiones 
de la presión esofágica aumentan progresivamente, como traducción de un incremento del trabajo respiratorio ${ }^{55,56}$. Acompañando al aumento del trabajo respiratorio, y como consecuencia directa de la mayor elastancia, estos pacientes desarrollan un patrón respiratorio rápido y superficial $^{57}$, y reclutan sus músculos respiratorios auxiliares ${ }^{58}$. El reclutamiento de los músculos inspiratorios auxiliares aparece precozmente y si la carga aumentada se mantiene en el tiempo se reclutan adicionalmente los músculos espiratorios, en un vano intento por apoyar la acción debilitada del diafragma ${ }^{58}$. La incapacidad del diafragma para asumir la carga inspiratoria que representa la desconexión ha sido bien documentada en la EPOC $^{59}$, pero puede verse afectada en otras enfermedades, ya sea por el daño asociado al reposo impuesto por la ventilación mecánica ${ }^{60,61}$ o como parte de las anormalidades neuromusculares del paciente crítico ${ }^{62,63}$. En estas últimas, se han documentado alteraciones electromiográficas del diafragma en pacientes con compromiso muscular periférico marcado ${ }^{64,65}$.

Cuando fracasa la desconexión también se afecta el intercambio pulmonar de gases. En los pacientes con EPOC se ha observado un empeoramiento de las relaciones como resultado de la redistribución de la perfusión hacia áreas de $\dot{V}_{\mathrm{A}} / \mathrm{Q}$ bajo $^{66}$. Esto explicaría la hipoxemia que acompaña a la desconexión fallida, mientras que la hipercapnia sería consecuencia de un aumento de la ventilación de espacio muerto, secundaria al patrón respiratorio rápido y superficial. En pacientes con insuficiencia respiratoria de otras causas, la hipoxemia probablemente sea consecuencia del colapso de unidades alveolares inestables, que incrementarían el cortocircuito intrapulmonar. El efecto inmediato de los trastornos del intercambio de gases es un incremento adicional de la demanda ventilatoria sobre un sistema respiratorio ya sobrecargado y débil.

En muchos pacientes la discontinuación de la ventilación mecánica puede fracasar por causas no respiratorias, como cardiopatía isquémica, valvular, o disfunción sistólica y/o diastólica de causas variadas. Estas condiciones pueden ser comorbilidades conocidas, pero a veces sólo se hacen evidentes durante la desconexión. La transición de la ventilación mecánica con presión positiva a la respiración espontánea se acompaña de un aumento del retorno venoso y de presiones intratorácicas negativas que incrementan la post carga ventricular y el consumo de oxígeno miocárdico. Esto explica que una disfunción miocárdica no conocida pueda manifestarse durante el intento de desconexión ${ }^{67,68}$. La sobrecar- ga cardiovascular se explica porque el gasto cardíaco debe invariablemente aumentar para cumplir con el aumento de la demanda metabólica que conlleva el proceso de desconexión. Cuando el sistema cardiovascular es sobrepasado por la demanda, el lactato sérico aumenta, la saturación venosa mixta de oxígeno $\left(\mathrm{SvO}_{2}\right)$ disminuye, y el $\mathrm{pH}$ de la mucosa gástrica se reduce durante la prueba de respiración espontánea ${ }^{69,70}$, hallazgos que en el contexto de una desconexión fallida sugieren isquemia tisular por desviación del gasto cardíaco hacia los músculos respiratorios.

La justificación de usar VNI para facilitar la desconexión de la ventilación mecánica radica en su capacidad para contrarrestar varios mecanismos fisiopatológicos asociados a su fracaso, particularmente el aumento de la carga de los músculos respiratorios. Este fundamento fisiológico ha sido confirmado en pacientes hipercápnicos con EPOC que habían fracasado a una prueba de ventilación espontánea en tubo $\mathrm{T}$ y que fueron sometidos sucesivamente a PSV invasiva a través del tubo endotraqueal y a PSV no invasiva después de proceder a la extubación $^{71}$. La PSV no invasiva después de la extubación fue capaz de mantener el intercambio gaseoso, el esfuerzo diafragmático y las propiedades mecánicas del sistema respiratorio en valores semejantes a los obtenidos durante la ventilación mecánica invasiva. Un efecto similar se observó también en pacientes sin EPOC que fueron extubados precozmente después de respirar espontáneamente en tubo $\mathrm{T}$ y asignados a VNI o $\mathrm{CPAP}^{72}$. Ambas modalidades mejoraron el patrón respiratorio, disminuyeron el trabajo respiratorio y redujeron el cortocircuito intrapulmonar en comparación con el período de respiración espontánea. La VNI también puede prevenir la disfunción miocárdica asociada a la desconexión, por mecanismos ya comentados a propósito del edema pulmonar agudo.

\section{Efectos beneficiosos no fisiológicos de la VNI}

Los resultados de los pacientes en los que fracasa la VNI y eventualmente requieren ventilación mecánica invasiva son comparables a los de aquellos que son conectados primariamente a ventilación mecánica, sin una prueba previa de $\mathrm{VNI}^{73,74}$. Por lo tanto, los beneficios clínicos asociados a la VNI en la insuficiencia respiratoria aguda parecen deberse principalmente al hecho de evitar la intubación endotraqueal. Los 
procedimientos invasivos, como los catéteres y tubos endotraqueales están entre los principales factores de riesgo para adquirir infecciones nosocomiales en las unidades de cuidados intensivos. En los pacientes ventilados mecánicamente el principal mecanismo etiopatogénico para adquirir una neumonía nosocomial es la aspiración de secreciones orofaríngeas colonizadas. En consecuencia, la VNI puede reducir el riesgo de tales infecciones debido a que la barrera glótica natural no se pierde, como ocurre después de una intubación, siendo las consecuencias clínicas una menor duración de la asistencia ventilatoria, la necesidad de sedación, y en último término, la duración de la estadía en la $\mathrm{UCI}^{75}$. La mayoría de los ensayos clínicos relacionados al uso de VNI ha mostrado una reducción en la frecuencia de todas las infecciones nosocomiales adquiridas en la $\mathrm{UCI}^{75-77}$, coincidiendo con una reducción sustancial en el número de procedimientos invasivos ${ }^{73,75,76,78,79}$.

\section{Conclusiones}

Existen fundamentos fisiológicos sólidos que apoyan el empleo clínico de la VNI durante la insuficiencia respiratoria aguda de distintas etiologías. En todas estas situaciones, la VNI puede reducir la carga de los músculos respiratorios, aliviar consecuentemente la disnea y mejorar el intercambio de gases. Por lo tanto, la VNI podría sustituir el empleo de ventilación mecánica convencional, evitando los riesgos que ella involucra y que han sido expuestos en el párrafo precedente.

Sin embargo, los estudios fisiológicos son de corta duración por razones prácticas y no necesariamente se ajustan a las condiciones clínicas del "mundo real". Así, el sustento físiológico parece sólido para la EPOC, pero ciertamente aquellos pacientes con una marcada HD, en estado de coma inducido por la hipercapnia, o con una acidosis respiratoria grave probablemente no toleren la VNI. Asimismo, los datos fisiológicos indican que la VNI no solamente apoya al sistema respiratorio en el EPA, sino que incluso la presión positiva intratorácica tiene un efecto benéfico sobre la función cardíaca. Sin embargo, esto no significa de manera alguna que el tratamiento médico convencional no sea suficiente en la mayoría de los pacientes. En la injuria pulmonar aguda, los hallazgos fisiológicos son aún más difíciles de trasladar a la clínica, ya que si algo caracteriza a este tipo de trastorno es su inestabilidad y la asociación fre- cuente con el compromiso de otros órganos, lo que implica riesgos de retrasar inapropiadamente la institución de ventilación mecánica convencional al emplear VNI. El escenario es tanto o más complejo en la desconexión de ventilación mecánica, pues en este caso la VNI es sólo una transición entre un tipo de soporte ventilatorio y otro. Asimismo, siendo las causas que motivan la ventilación mecánica muy heterogéneas, y múltiples los mecanismos que hacer fracasar la desconexión, los efectos fisiológicos benéficos son de difícil interpretación.

\section{Bibliografía}

1.- BHOWMIK A, SEEMUNGAL T A R, SAPSFORD R J, WEDZICHA J A. Relation of sputum inflammatory markers to symptoms and lung function changes in COPD exacerbations. Thorax 2000; 55: 114-20.

2.- PAPI A, BELLETTATO C M, BRACCIONI F, ROMAGNOLI M, CASOLARI P, CARAMORI G, et al. Infections and airway inflammation in chronic obstructive pulmonary disease severe exacerbations. Am J Respir Crit Care Med 2006; 173: 1114-21.

3.- ALVISI V, ROMANELLO A, BADET M, GAILLARD S, PHILIT F, GUERIN C. Time course of expiratory flow limitation in COPD patients during acute respiratory failure requiring mechanical ventilation. Chest. 2003; 123: 1625-32.

4.- LISBOA C, DÍAZ O, BORZONE G. The ventilatory factors in exercise performance in patients with chronic obstructive pulmonary disease. In: Amid Q, Shannon J, Martín J, eds. Physiological Basis of Respiratory Disease. London. BC Decker Inc. Publisher 2005; 541-6.

5.- O'DONNELL D E, PARKER C M. COPD exacerbations * 3: Pathophysiology. Thorax 2006; 61: 35461 .

6.- ROUSSOS C, KOUTSOUKOU A. Respiratory failure. Eur Respir J 2003; 22 (suppl): 3S-14.

7.- PARE P, BROOKS L, BATES J, LAWSON L, NELEMS J, WRIGHT J, et al. Exponential analysis of the lung pressure-volume curve as a predictor of pulmonary emphysema. Am Rev Respir Dis 1982; 126 : 54-61.

8.- HALUSZKA J, CHARTRAND D, GRASSINO A, MILIC-EMILI J. Intrinsic PEEP and arterial $\mathrm{PCO}_{2}$ in stable patients with chronic obstructive pulmonary disease. Am Rev Respir Dis 1990; 141 (5 Pt 1): 1194 7.

9.- COUSSA M L, GUERIN C, EISSA N T, CORBEIL C, CHASSE M, BRAIDY J, et al. Partitioning of work of breathing in mechanically ventilated COPD patients. J Appl Physiol 1993; 75: 1711-9.

10.- OROZCO-LEVI M, GEA J, LLORETA J L, FELEZ M, MINGUELLA J, SERRANO S, et al. Subcellular adaptation of the human diaphragm in chronic obstructive pulmonary disease. Eur Respir J 1999; 13: 371-8.

11.- LEVINE S, KAISER L, LEFEROVICH J, TIKUNOV B. Cellular adaptations in the diaphragm in chronic obstructive pulmonary disease. N Engl J Med 1997; 337: $1799-806$. 
12.- SIMILOWSKI T, YAN S, GAUTHIER A P, MACKLEM P T, BELLEMARE F. Contractile properties of the human diaphragm during chronic hyperinflation. N Engl J Med 1991; 325: 917-23.

13.- POLKEY M I, KYROUSSIS D, HAMNEGARD C H, MILLS G H, GREEN M, MOXHAM J. Diaphragm strength in chronic obstructive pulmonary disease. Am. J. Respir. Crit. Care Med 1996; 154: 1310-7.

14.- OROZCO-LEVI M, LLORETA J, MINGUELLA J, SERRANO S, BROQUETAS J M, GEA J. Injury of the human diaphragm associated with exertion and chronic obstructive pulmonary disease. Am J Respir Crit Care Med 2001; 164: 1734-9.

15.- OTTENHEIJM C A C, HEUNKS L M A, DEKHUIJZEN P N R. Diaphragm muscle fiber dysfunction in chronic obstructive pulmonary disease: Toward a pathophysiological concept. Am J Respir Crit Care Med 2007; 175: $1233-40$.

16.- DE TROYER A, LEEPER J, MCKENZIE D, GANDEVIA S. Neural drive to the diaphragm in patients with severe COPD. Am J Respir Crit Care Med 1997; 155: 1335-40.

17.- SINDERBY C, SPAHIJA J, BECK J, KAMINSKI D, YAN S, COMTOIS N, et al. Diaphragm activation during exercise in chronic obstructive pulmonary disease. Am J Respir Crit Care Med 2001; 163: 163741.

18.- O'DONNELL D, BERTLEY J, CHAU L, WEBB K. Qualitative aspects of exertional breathlessness in chronic airflow limitation: pathophysiologic mechanisms. Am J Respir Crit Care Med 1997; 155: 109-15.

19.- O'DONNELL D E, REVILL S M, WEBB K A. Dynamic hyperinflation and exercise intolerance in chronic obstructive pulmonary disease. Am J Respir Crit Care Med 2001; 164: 770-7.

20.- LOUGHEED D, WEBB K, O’DONNELL D. Breathlessness during induced lung hyperinflation in asthma: the role of the inspiratory threshold load. Am J Respir Crit Care Med 1995; 152: 911-20.

21.- BARBERA J A, ROCA J, FERRER A, FELEZ M A, DÍAZ O, ROGER N, et al. Mechanisms of worsening gas exchange during acute exacerbations of chronic obstructive pulmonary disease. Eur Respir J 1997; 10 : 1285-91.

22.- BROCHARD L, ISABEY D, PIQUET J, AMARO P, MANCEBO J, MESSADI A A, et al. Reversal of acute exacerbations of chronic obstructive lung disease by inspiratory assistance with a face mask. N Engl J Med 1990; 323:1523-30.

23.- APPENDINI L, PATESSIO A, ZANABONI S, CARONE M, GUKOV B, DONNER C F, et al. Physiologic effects of positive end-expiratory pressure and mask pressure support during exacerbations of chronic obstructive pulmonary disease. Am J Respir Crit Care Med 1994; 149:1069-76.

24.- CARREY Z, GOTTFRIED S B, LEVY R D. Ventilatory muscle support in respiratory failure with nasal positive pressure ventilation. Chest 1990; 97: 150-8.

25.- DÍAZ O, IGLESIA R, FERRER M, ZAVALA E, SANTOS C, WAGNER PETER D, et al. Effects of noninvasive ventilation on pulmonary gas exchange and hemodynamics during acute hypercapnic exacerbations of chronic obstructive pulmonary disease. Am J Respir Crit Care Med 1997; 156: 18405.

26.- STAUB N C. Pulmonary edema. Physiol Rev 1974; 54: $678-811$.
27.- MATTHAY M A, FOLKESSON H G, CLERICI C. Lung epithelial fluid transport and the resolution of pulmonary edema. Physiol Rev 2002; 82: 569-600.

28.- SHARP J T, GRIFFITH G T, BUNNELL I L, GREENE D G. Ventilatory mechanics in pulmonary edema in man. J Clin Invest 1958; 37: 111-7.

29.- NAVA S, LAROVERE M, FANFULLA F, NAVALESI P, DELMASTRO M, MORTARA A. Orthopnea and inspiratory effort in chronic heart failure patients. Respir Med 2003; 97: 647-53.

30.- YAP J C H, MOORE D M, CLELAND J G F, PRIDE $\mathrm{N}$ B. Effect of supine posture on respiratory mechanics in chronic left ventricular failure. Am J Respir Crit Care Med 2000; 162: 1285-91.

31.- HOGG J C, AGARAWAL J B, GARDINER A J, PALMER W H, MACKLEM P T. Distribution of airway resistance with developing pulmonary edema in dogs. J Appl Physiol 1972; 32: 20-4.

32.- MICHEL R P, ZOCCHI L, ROSSI A, CARDINAL G A, PLOY-SONG-SANG Y, POULSEN R S, et al. Does interstitial lung edema compress airways and arteries? A morphometric study. J Appl Physiol 1987; 62: 10815.

33.- DEPEURSINGE F, DEPEURSINGE C, BOUTALEB A, FEIHL F, PERRET C. Respiratory system impedance in patients with acute left ventricular failure: pathophysiology and clinical interest. Circulation 1986; 73: 386-95.

34.- DUGUET A, TANTUCCI C, LOZINGUEZ O, ISNARD R, THOMAS D, ZELTER M, et al. Expiratory flow limitation as a determinant of orthopnea in acute left heart failure. J Am Coll Cardiol 2000; 35: 690-700.

35.- CHADDA K, ANNANE D, HART N, GAJDOS P, RAPHAEL J, LOFASO F. Cardiac and respiratory effects of continuous positive airway pressure and noninvasive ventilation in acute cardiac pulmonary edema. Crit Care Med 2002; 30: 2457-61.

36.- GEHLBACH B K, GEPPERT E. The pulmonary manifestations of left heart failure. Chest 2004; 125 : 669-82.

37.- NOBLE W H, KAY J C, OBDRZALEK J. Lung mechanics in hypervolemic pulmonary edema. J Appl Physiol 1975; 38: 681-7.

38.- BUDA A, PINSKY M, INGELS N, DAUGHTERS G, STINSON E, ALDERMAN E. Effect of intrathoracic pressure on left ventricular performance. N Engl J Med 1979; 301: 453-9.

39.- NAUGHTON M T, RAHMAN M A, HARA K, FLORAS J S, BRADLEY T D. Effect of continuous positive airway pressure on intrathoracic and left ventricular transmural pressures in patients with congestive heart failure. Circulation 1995; 91: 172531 .

40.- LENIQUE F, HABIS M, LOFASO F, DUBOIS-RANDE J L, HARF A, BROCHARD L. Ventilatory and hemodynamic effects of continuous positive airway pressure in left heart failure. Am J Respir Crit Care Med 1997; 155: 500-5.

41.- BENDJELID K, SCHUTZ N, SUTER P M, FOURNIER G, JACQUES D, FAREH S, et al. Does continuous positive airway pressure by face mask improve patients with acute cardiogenic pulmonary edema due to left ventricular diastolic dysfunction? Chest 2005; 127: 1053-8.

42.- FESSLER H E, BROWER R G, WISE R A, PERMUTT S. Effects of systolic and diastolic positive pleural pressure pulses with altered cardiac contractility. J Appl 
Physiol 1992; 73: 498-505.

43.- FESSLER H E, BROWER R G, WISE R A, PERMUTT S. Mechanism of reduced LV afterload by systolic and diastolic positive pleural pressure. J Appl Physiol 1988; 65: $1244-50$.

44.- MASIP J, ROQUE M, SÁNCHEZ B, FERNÁNDEZ R, SUBIRANA M, EXPOSITO J A. Noninvasive ventilation in acute cardiogenic pulmonary edema: Systematic review and meta-analysis. JAMA 2005; 294: 3124 30.

45.- WINCK J, AZEVEDO L, COSTA-PEREIRA A, ANTONELLI M, WYATT J. Efficacy and safety of non-invasive ventilation in the treatment of acute cardiogenic pulmonary edema - a systematic review and meta-analysis. Critical Care 2006; 10: R69.

46.- MEHTA S, JAY G, WOOLARD R, HIPONA R, CONNOLLY E, CIMINI D, et al. Randomized, prospective trial of bilevel versus continuous positive airway pressure in acute pulmonary edema. Crit Care Med 1997; 25: 620-8.

47.- NAVA S, CARBONE G, DIBATTISTA N, BELLONE A, BAIARDI P, COSENTINI R, et al. Noninvasive ventilation in cardiogenic pulmonary edema: A multicenter randomized trial. Am J Respir Crit Care Med 2003; 168: 1432-7.

48.- LEAVER S K, EVANS T W. Acute respiratory distress syndrome. BMJ 2007; 335: 389-94.

49.- DANTZKER D, BROOK C, DEHART P, LYNCH J, WEG J. Ventilation-perfusion distributions in the adult respiratory distress syndrome. Am Rev Respir Dis 1979; 120: $1039-52$.

50.- PELOSI P, D'ONOFRIO D, CHIUMELLO D, PAOLO S, CHIARA G, CAPELOZZI V L, et al. Pulmonary and extrapulmonary acute respiratory distress syndrome are different. Eur Respir J 2003; 22 (suppl): 48s-56.

51.- L'HER E, DEYE N, LELLOUCHE F, TAILLE S, DEMOULE A, FRATICELLI A, et al. Physiologic effects of noninvasive ventilation during acute lung injury. Am J Respir Crit Care Med 2005; 172: 1112-8.

52.- BOLES J M, BION J, CONNORS A, HERRIDGE M, MARSH B, MELOT C, et al. Weaning from mechanical ventilation. Eur Respir J 2007; 29: 1033-56.

53.- STRAUS C, LOUIS B, ISABEY D, LEMAIRE F, HARF A, BROCHARD L. Contribution of the endotracheal tube and the upper airway to breathing workload. Am J Respir Crit Care Med 1998; 157: 23-30.

54.- SALAM A, TILLUCKDHARRY L, AMOATENGADJEPONG Y, MANTHOUS C A. Neurologic status, cough, secretions and extubation outcomes. Intensive Care Medicine 2004; 30: 1334-9.

55.- JUBRAN A, TOBIN M. Pathophysiologic basis of acute respiratory distress in patients who fail a trial of weaning from mechanical ventilation. Am J Respir Crit Care Med 1997; 155: 906-15.

56.- JUBRAN A, GRANT B J B, LAGHI F, PARTHASARATHY S, TOBIN M J. Weaning prediction: Esophageal pressure monitoring complements readiness testing. Am J Respir Crit Care Med 2005; 171: 1252 9.

57.- TOBIN M, PÉREZ W, GUENTHER S, SEMMES B, MADOR M, ALLEN S, et al. The pattern of breathing during successful and unsuccessful trials of weaning from mechanical ventilation. Am Rev Respir Dis 1986; 134: 1111-8.

58.- PARTHASARATHY S, JUBRAN A, LAGHI F, TOBIN M J. Sternomastoid, rib cage, and expiratory muscle activity during weaning failure. J Appl Physiol 2007;
103: $140-7$

59.- VASSILAKOPOULOS T, ZAKYNTHINOS S, ROUSSOS C. The tension-time index and the frequency/ tidal volume ratio are the major pathophysiologic determinants of weaning failure and success. Am J Respir Crit Care Med 1998; 158: 378-85.

60.- VASSILAKOPOULOS T, PETROF B J. Ventilatorinduced diaphragmatic dysfunction. Am J Respir Crit Care Med 2004; 169: 336-41.

61.- LEVINE S, NGUYEN T, TAYLOR N, FRISCIA M E, BUDAK M T, ROTHENBERG P, et al. Rapid disuse atrophy of diaphragm fibers in mechanically ventilated humans. N Engl J Med 2008; 358: 1327-35.

62.- DE JONGHE B, BASTUJI-GARIN S, SHARSHAR T, OUTIN H, BROCHARD L. Does ICU-acquired paresis lengthen weaning from mechanical ventilation? Intensive Care Medicine 2004; 30: 1117-21.

63.- SCHWEICKERT W D, HALL J. ICU-acquired weakness. Chest 2007; 131: 1541-9.

64.- WITT N, ZOCHODNE D, BOLTON C, GRAND' MAISON F, WELLS G, YOUNG G, et al. Peripheral nerve function in sepsis and multiple organ failure. Chest 1991; 99: 176-84.

65.- MAHER J, RUTLEDGE F, REMTULLA H, PARKES A, BERNARDI L, CF. B. Neuromuscular disorders associated with failure to wean from the ventilator. Intensive Care Med 1995; 21: 737-43.

66.- TORRES A, REYES A, ROCA J, WAGNER P, RODRÍGUEZ-ROISIN R. Ventilation-perfusion mismatching in chronic obstructive pulmonary disease during ventilator weaning. Am Rev Respir Dis 1989; 140: $1246-50$.

67.- LEMAIRE F, TEBOUL J, CINOTTI L, GIOTTO G, ABROUK F, STEG G, et al. Acute left ventricular dysfunction during unsuccessful weaning from mechanical ventilation. Anesthesiology 1988; 69: 171-9.

68.- PINSKY M R, SUMMER W R, WISE R A, PERMUTT S, BROMBERGER-BARNEA B. Augmentation of cardiac function by elevation of intrathoracic pressure. J Appl Physiol 1983; 54: 950-5.

69.- JUBRAN A, MATHRU M, DRIES D, TOBIN MARTÍN J. Continuous recordings of mixed venous oxygen saturation during weaning from mechanical ventilation and the ramifications thereof. Am J Respir Crit Care Med 1998; 158: 1763-9.

70.- MALDONADO A, BAUER T T, FERRER M, HERNÁNDEZ C, ARANCIBIA F, RODRÍGUEZROISIN $\mathrm{R}$, et al. Capnometric recirculation gas tonometry and weaning from mechanical ventilation. Am J Respir Crit Care Med 2000; 161: 171-6.

71.- VITACCA M, AMBROSINO N, CLINI E, PORTA R, RAMPULLA C, LANINI B, et al. Physiological response to pressure support ventilation delivered before and after extubation in patients not capable of totally spontaneous autonomous breathing. Am J Respir Crit Care Med 2001; 164: 638-41.

72.- KILGER E, BRIEGEL J, HALLER M, FREY L, SCHELLING G, STOLL C, et al. Effects of noninvasive positive pressure ventilatory support in nonCOPD patients with acute respiratory insufficiency after early extubation. Intensive Care Medicine 1999; 25: $1374-80$

73.- BROCHARD L, MANCEBO J, WYSOCKI M, LOFASO F, CONTI G, RAUSS A, et al. Noninvasive ventilation for acute exacerbations of chronic obstructive pulmonary disease. N Engl J Med 1995; 333: 817-22.

74.- CARLUCCI A, RICHARD J-C, WYSOCKI M, LEPAGE E, BROCHARD L AND THE COLLABORATIVE 
GROUP ON MECHANICAL VENTILATION. Noninvasive versus conventional mechanical ventilation. An epidemiologic survey. Am J Respir Crit Care Med 2001; 163: 874-80.

75.- GIROU E, BRUN-BUISSON C, TAILLE S, LEMAIRE F, BROCHARD L. Secular trends in nosocomial infections and mortality associated with noninvasive ventilation in patients with exacerbation of COPD and pulmonary edema. JAMA 2003; 290: 2985-91.

76.- GIROU E, SCHORTGEN F, DELCLAUX C, BRUNBUISSON C, BLOT F, LEFORT Y, et al Association of noninvasive ventilation with nosocomial infections and survival in critically ill patients. JAMA 2000; 284 :
2361-7.

77.- BROCHARD L. Noninvasive ventilation for acute respiratory failure. JAMA 2002; 288: 932-5.

78.- NAVA S, EVANGELISTI I, RAMPULLA C, COMPAGNONI M L, FRACCHIA C, RUBINI F. Human and financial costs of noninvasive mechanical ventilation in patients affected by COPD and acute respiratory failure. Chest 1997; 111: 1631-8.

79.- ANTONELLI M, CONTI G, ROCCO M, BUFI M, DE BLASI R A, VIVINO G, et al. A comparison of noninvasive positive-pressure ventilation and conventional mechanical ventilation in patients with acute respiratory failure. N Engl J Med 1998; 339: 429-35. 